\title{
Setorização de rotas de coleta de resíduos sólidos domiciliares por técnicas multivariadas: estudo de caso da cidade do Recife, Brasil
}

\author{
Sectorization of solid household waste collection routes by multivariate \\ techniques: a case study of the city of Recife, Brazil
}
Rodrigo Cândido Passos da Silva'* ${ }^{1}$, Amanda Rodrigues Santos Costa' ${ }^{(}$, Soraya Giovanetti El-Deir ${ }^{2}$ (†, José Fernando Thomé Jucá' ${ }^{\circledR}$

\section{RESUMO}

Buscar soluções para a problemática dos resíduos sólidos domiciliares (RSD) é um dos desafios enfrentados pelos gestores municipais. Diante do cenário ascendente de geração desses materiais, principalmente nos centros urbanos, a implantação de um modelo integrado eadequado à realidade da municipalidade torna-se imprescindivel. Nesse sentido, a setorização de rotas de coleta a partir da utilização de técnicas multivariadas pode auxiliar o gestor na proposição de modelos setoriais de resíduos sólidos que contemplem as particularidades socioeconômicas das áreas estudadas. Desse modo, o presente artigo utiliza as técnicas multivariadas de análise de componentes principais, para avaliar a correlação entre população, renda, geração e composição gravimétrica dos resíduos, elencando, entre esses indicadores, os mais representativos; e a análise de agrupamento hierárquico, para setorizar 31 rotas de coleta em clusters por similaridade. Verificou-se que a população, a renda domiciliar e per capita, a geração de resíduos per capita e os resíduos recicláveis, alimentares e putresciveis foram bem representados no estudo. A renda e a geração per capita apresentaram forte correlação positiva. A setorização das rotas gerou dois grupos: $A$ e $B$, que resultaram em cinco subgrupos por similaridade: $A 1, A 2$, B1, B2 e B3. Os subgrupos A1 e A2 contemplaram os setores de classe baixa, ao passo que os clusters B1, B2 e B3 compreenderam os setores de classe alta, média e comercial, respectivamente. A setorização de rotas mediante o grau de similaridade apresenta-se como etapa basilar para a proposição de modelos setoriais de RSD que busquem o entendimento das particularidades elencadas. Palavras-chave: gestão de resíduos sólidos; setores de coleta; indicadores gerenciais; análise de componente principal; análise de agrupamento hierárquico.

\begin{abstract}
Finding solutions to the problem of household solid waste (HSW) is one of the challenges faced by municipal managers. In view of the upward scenario of generation of these materials, especially in urban centers, the implementation of an integrated model and adequate to the reality of society is essential. In this sense, the sectorization of collection routes from the use of multivariate techniques can assist the manager in proposing solid waste sector models that take into account the socioeconomic particularities of the studied areas. In this way, the present article uses the multivariate techniques of Analysis of Principal Components to evaluate the correlation of population, income, generation, and gravimetric composition of the residues, listing among these most representative indicators; and the Hierarchical Grouping Analysis to segment 31 collection routes into clusters by similarity. Population, household and per capita income, per capita waste production and recyclable, food and putrescible residues were well represented in the study. Income and per capita production showed a strong positive correlation. The segmentation of the routes generated two groups: $A$ and $B$ which resulted in five subgroups by similarity: $A 1, A 2, B 1, B 2$, and $B 3$. The $A 1$ and A2 subgroups comprised the lower class sectors, while the B1, B2, and B3 clusters comprised the upper, middle, and commercial sectors, respectively. The sectorization of routes through the degree of similarity presents itself as the basic step for the proposition of HSW sectorial models that seek to understand the particularities listed.
\end{abstract}

Keywords: solid waste management; collection sectors; management indicators; principal component analysis; hierarchical grouping analysis.

\section{INTRODUÇÃO}

Nos últimos anos, a aceleração do crescimento populacional, principalmente nos centros urbanos, aliada à ascensão da renda da população e ao aumento de produção e consumo vigentes, tem provocado elevados índices de geração de resíduos sólidos urbanos (RSU). Esse aumento substancial torna-se um entrave para os gestores municipais no tocante

Universidade Federal de Pernambuco - Recife (PE), Brasil.

¿Universidade Federal Rural de Pernambuco - Recife (PE), Brasil.

*Autor correspondente: rcpassos13@gmail.com

Recebido: 21/05/2018 - Aceito: 22/08/2019 - Reg. ABES: 200205 
à oferta dos serviços de coleta, transporte e disposição final desses materiais. A baixa efetividade desse serviço acarreta danos visuais à cidade, agravando o problema de poluição ambiental, bem como compromete a qualidade de vida dos moradores urbanos. De acordo com o SNIS (2017), publicado em 2019, a quantidade de resíduos sólidos domiciliares e públicos coletados no Brasil em 2017 foi de 60,6 milhões de $\mathrm{t}$. Esse valor foi acrescido em 2,89\%, quando comparado com o ano de 2016. Em Recife, esse quantitativo foi de aproximadamente $825,4 \mathrm{mil} \mathrm{t}$ em 2017 (SNIS, 2017). Essa problemática exige soluções, haja vista que interfere nas dimensões econômica, ambiental, social e de saúde pública, além de prejudicar o desenvolvimento urbano sustentável do país.

Nesse sentido, pesquisas voltadas para essa temática têm atraído a atenção de pesquisadores nos últimos anos. De acordo com Purcell e Magette (2009), a inserção de técnicas estatísticas tem se mostrado relevante para auxiliar a municipalidade no planejamento e na gestão dos resíduos sólidos. As técnicas multivariadas se destacam por possibilitar a análise simultânea de vários indicadores e apontar possíveis estratégias gerenciais para os gestores públicos. Vários trabalhos foram publicados nessa perspectiva. Bari, Mata e Wanderley (2014) utilizaram a análise fatorial para determinar o grau de adequação do gerenciamento dos RSU em 27 estados do Brasil. Lenz et al. (2016) usaram modelos de regressão por mínimos quadrados parciais (PLS) para monitorar o lixiviado de aterros sanitários. Chu et al. (2016) empregaram a regressão múltipla para desenvolver um modelo estatístico que apontava as variáveis de influência na geração de RSU da China. Sel et al. (2016) utilizaram a regressão linear múltipla (MLR) para gerar um modelo de previsão do potencial bioquímico de metano (BMP) com base na composição dos resíduos dispostos no aterro de Istambul, na Turquia. Já Chassaigne e Pinto (2014) usaram a regressão múltipla para elaborar um modelo de geração de resíduos sólidos domiciliares (RSD) para 175 municípios da Venezuela.

Além das técnicas de regressão, outras ferramentas multivariadas vêm sendo aplicadas para tal fim, assim como a análise de componentes principais (PCA) e a análise de agrupamento hierárquico (análise por cluster). Caribé (2015) utilizou a PCA para avaliar o comportamento geomecânico de uma célula experimental. Fudala-Ksiazek et al. (2016) empregaram a PCA para correlacionar características físico-químicas e microbiológicas do lixiviado gerado por um biodigestor. Ni, Liu e Zhang (2017) analisaram a interferência de indicadores químicos e espectroscópicos em lixiviados anaeróbicos com base na PCA. Schirmer et al. (2016) utilizaram a PCA para correlacionar o volume de biogás gerado com as características físico-químicas dos resíduos aterrados, ao passo que Costa (2017) utilizou a PCA e a análise por cluster para avaliar a inter-relação de indicadores de sustentabilidade nos setores de coleta de resíduos sólidos da cidade do Recife, Brasil. Rego et al. (2013) utilizaram a análise por cluster para agrupar alguns indicadores de saneamento ambiental, possibilitando a identificação de áreas prioritárias para intervenções e alocação de recursos, já Sulkowski e White (2016) utilizaram a análise por cluster para agrupar 61 países por similaridade mediante indicadores de felicidade, desenvolvimento, renda e emissão de carbono. Agovino, Ferrara e Garofalo (2016) agruparam, por meio da análise por cluster combinada com a análise espacial exploratória (ESDA), 103 províncias italianas por similaridade, sendo que Lima et al. (2017) agruparam 116 países por similaridade com base em indicadores de competitividade e desenvolvimento sustentável. Tais informações ratificam que esse método está tendo usos diversos na busca por compreender a realidade de dados que apresentam grau de similaridade.

De forma a subsidiar a tomada de decisão da municipalidade e auxiliar na gestão dos RSD, o presente trabalho teve o objetivo de empregar técnicas multivariadas para setorizar rotas de coleta domiciliar em cluster por similaridade, com base em indicadores definidores de 31 rotas de coleta da cidade do Recife. Pretende-se que a setorização dessas rotas auxilie os gestores municipais na proposição de modelos gerenciais heterogêneos, com foco nas particularidades locais, conforme preconiza a Política Nacional de Resíduos Sólidos (PNRS) (BRASIL, 2010).

\section{METODOLOGIA}

\section{Área de estudo}

O estudo foi realizado na cidade do Recife, por apresentar instrumento legal já elaborado, como a Política Estadual de Resíduos Sólidos (PERS), ancorada na Lei nº 14.236 (PERNAMBUCO, 2010), Plano de Gestão de Resíduos Sólidos em fase de elaboração e pela facilidade quanto ao acesso às informações. Recife é a capital do estado de Pernambuco, está localizada na Região Nordeste do Brasil (Figura 1), sendo a terceira maior metrópole regional. $\mathrm{O}$ município está situado a $7 \mathrm{~m}$ de altitude e posicionado nas coordenadas geográficas de $8^{\circ} 3^{\prime} 15^{\prime \prime}$ sul e $34^{\circ} 52^{\prime} 53^{\prime \prime}$ oeste. Com área de $218,435 \mathrm{~km}^{2}$, está dividida em 6 regiões político-administrativas (RPAs), compreendendo 94 bairros. Tem população estimada de 1.625.583 habitantes, densidade demográfica de 7.441,95 hab. $\mathrm{km}^{-2}$, produto interno bruto (PIB) per capita de $\mathrm{R} \$ 31.513,07 \mathrm{e}$ índice de desenvolvimento urbano municipal (IDHM) de 0,772 para o ano de 2016 (IBGE, 2016).

A Secretaria de Infraestrutura e Serviços Urbanos é o órgão responsável pelo sistema de manutenção e limpeza urbana da cidade, sendo a incumbência gerencial atribuída à Empresa de Manutenção e Limpeza Urbana (Emlurb). Em 2013, foram coletadas 548.919,10 t de RSU, com valor per capita de $0,94 \mathrm{~kg} \cdot \mathrm{hab}^{-1}$. $\mathrm{dia}^{-1}$, sendo $72,9 \%$ correspondentes aos resíduos orgânicos, $22 \%$ a fração reciclável e 5,1\% aos outros resíduos (SECID, 2015). A coleta de resíduos convencional é predominante na cidade, com taxa de cobertura em torno de $100 \%$. No entanto, a coleta seletiva da fração seca reciclável é limitada, correspondendo a 0,5\% da 
coleta domiciliar no ano de 2016, além de não existir coleta diferenciada para os resíduos orgânicos domiciliares. A Central de Tratamento de Resíduos (CTR Candeias) é o principal local de destinação final, dada a coleta convencional de RSD do município.

O estudo procedeu mediante a definição de rotas de coleta de RSD presentes no mapa fornecido pela Vital Engenharia S/A, concessionária detentora de $70 \%$ da coleta convencional domiciliar da cidade, correspondendo à prestação do serviço em 81 bairros do município. As rotas ou setores de coleta são microáreas que compreendem bairros, de forma parcial ou total, pelos quais passam caminhões coletores que realizam a coleta e limpeza urbana.

As rotas foram selecionadas por meio de uma amostragem estratificada, com base nos seguintes critérios: bairros inseridos na rota, concentração populacional, densidade demográfica, número de domicílios, nível econômico, geração e composição dos resíduos, presença de estabelecimentos comerciais e/ou domiciliares (Tabela 1). Sendo assim, foram selecionadas 31 rotas, correspondendo a aproximadamente $30 \%$ da população. Esses dados são oriundos de consultoria prestada pelo grupo de resíduos sólidos (GRS), da Universidade Federal de Pernambuco (UFPE), à Vital Engenharia, nos meses de dezembro/2013 a março/2014.

As informações relativas a bairros inseridos nas rotas de coleta, população, densidade demográfica, número de domicílios e renda total e per capita foram obtidas em Jucá et al. (2014), com base nos dados censitários do Instituto Brasileiro de Geografia e Estatística (IBGE, 2010). Os resultados alusivos à renda média domiciliar foram analisados e agrupados em classes econômicas, por meio da Tabela de Classificação Social da Secretaria de Assuntos Estratégicos da Presidência da República do Brasil (Tabela 2) (Brasil, 2012). Os dados de geração média e per capita de resíduos nas rotas, referentes ao ano de 2013, foram fornecidos pela Vital Engenharia. A geração de resíduos correspondeu à quantidade média mensal de RSD coletado pelo sistema de coleta convencional, o qual foi integralmente destinado ao Aterro

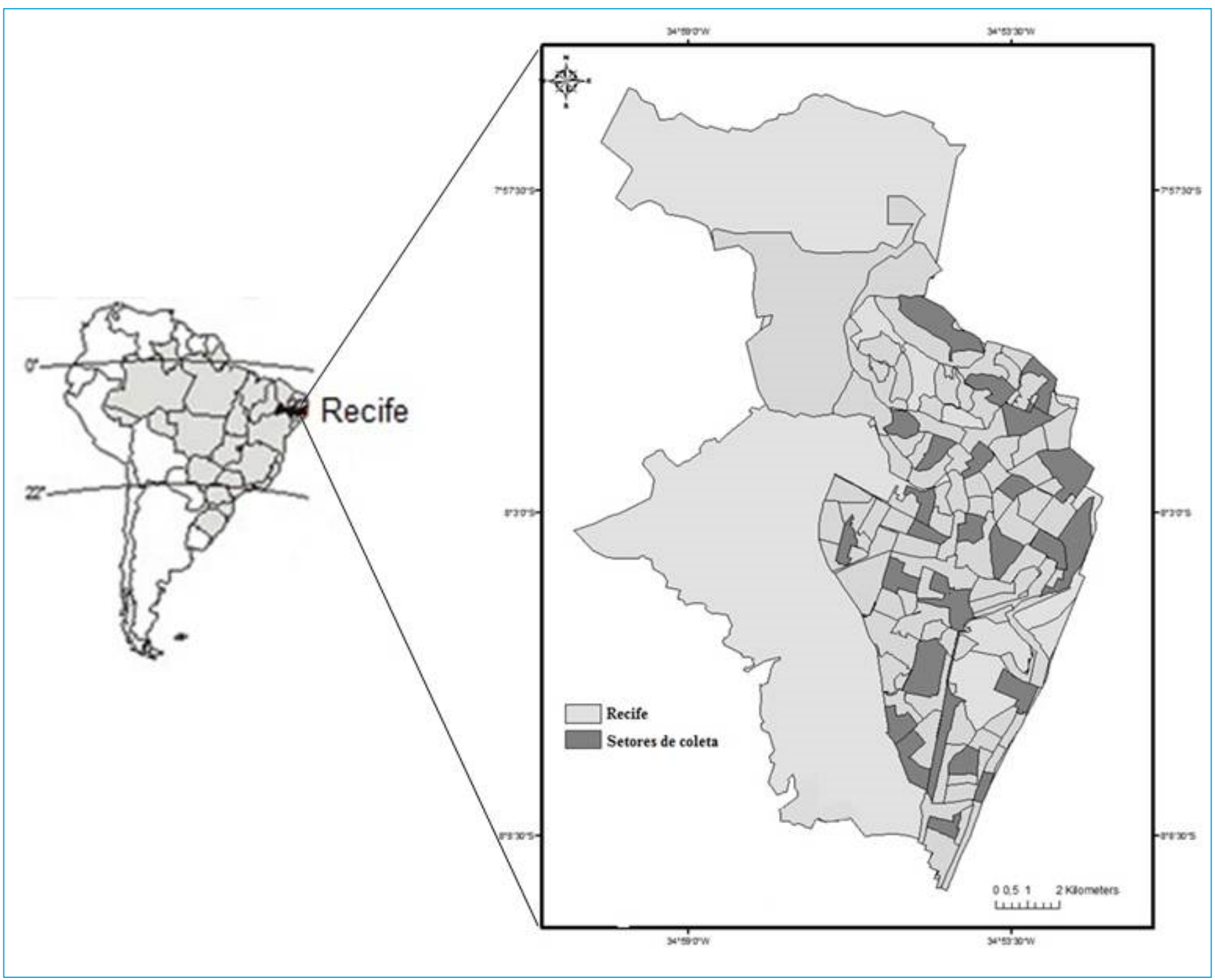

Figura 1 - Localização geográfica do Recife e identificação das 31 rotas de coleta de resíduos sólidos domiciliares. 
Tabela 1 - Características das 31 rotas de coleta de resíduos sólidos domiciliares selecionados para o estudo.

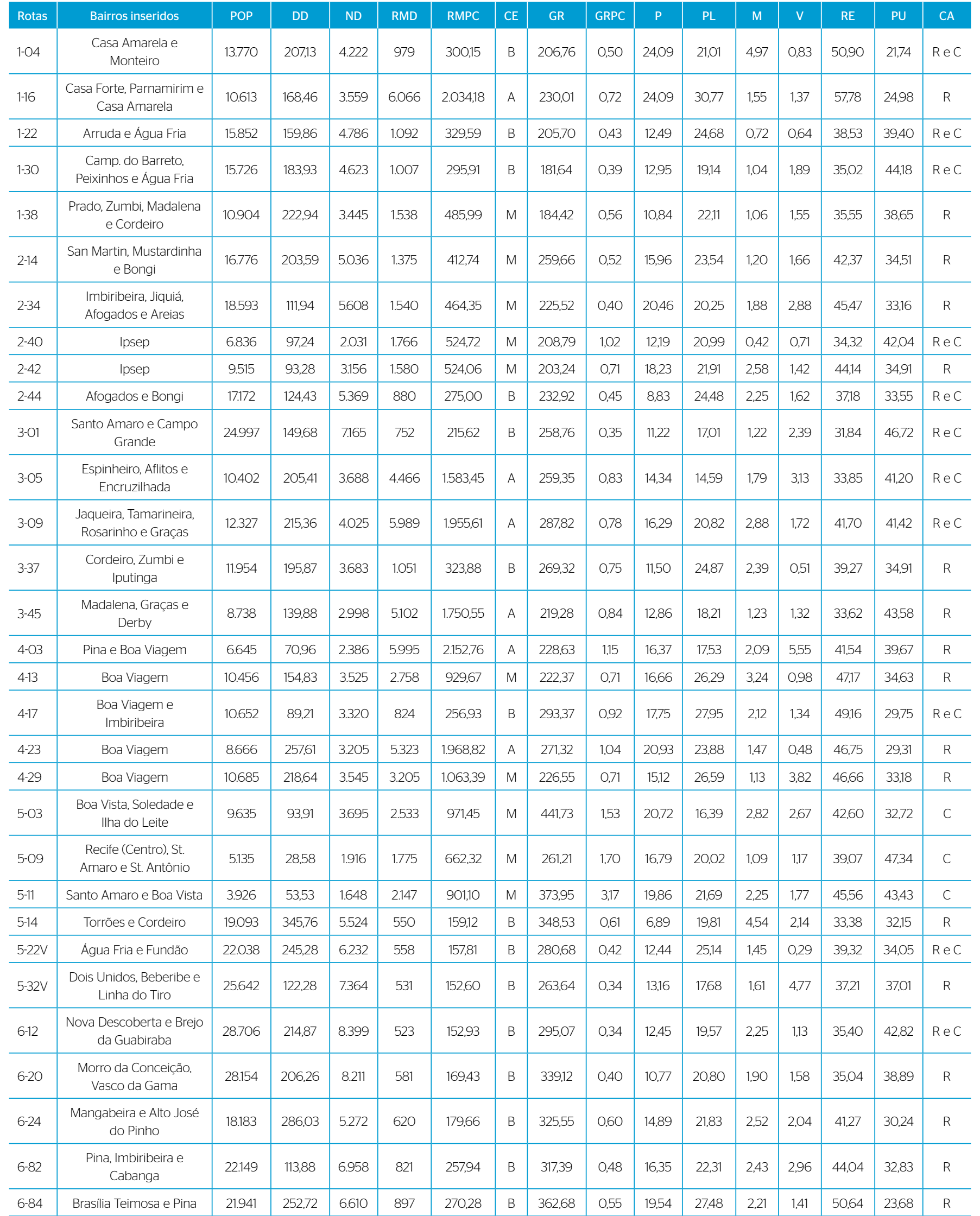

POP: população (hab.); DD: densidade demográfica (hab.ha'); ND: número de domicílios; RMD: renda média domiciliar (R\$.mês'); RMPC: renda média per capita (R\$.hab.'mês'1); CE: classe econômica; A: alta; M: média; B: baixa; GR: geração de resíduos (t.mês'1); GRPC: geração de resíduos per capita (kg.hab:'.dia'1); P: papel (\%); PL: plástico (\%); M: metal (\%); V: vidro (\%); RE: recicláveis (\%); PU: putrescíveis (\%); CA: características; R: residencial; C: comercial. 
Sanitário CTR Candeias. A geração per capita (kg.hab..$^{-1} \cdot$ dia $\left.^{-1}\right)$ foi calculada pela Equação 1:

$$
G R=\frac{G P R C}{P O P}
$$

Na qual:

GRPC = geração de resíduos per capita $\left(\mathrm{kg} \cdot h \mathrm{hab} \cdot{ }^{-1} \cdot \operatorname{dia}^{-1}\right)$;

$\mathrm{GR}=$ geração média mensal de resíduos $\left(\mathrm{kg} \cdot \mathrm{mês}^{-1}\right)$;

POP = população estimada na rota para o ano de 2013, com base nos dados do Censo 2010 (IBGE, 2010).

A composição gravimétrica das 31 rotas de RSD ocorreu nas proximidades da área de descarte final da CTR Candeias durante os meses de novembro e dezembro de 2013, nos turnos da manhã e da tarde. A amostragem dos resíduos seguiu a técnica de quarteamento, com base na metodologia da NBR 10.007 (ABNT, 2004). Dessa forma, os resíduos (em torno de $12 \mathrm{t}$ ) foram descarregados em uma área pavimentada e protegida com lona com o auxílio de um caminhão compactador, tendo sido previamente homogeneizados por uma retroescavadeira hidráulica, separados em quatro partes iguais com cerca de 2,0 t (primeiro quarteamento) e selecionados aleatoriamente em duas partes. Esse procedimento foi realizado novamente resultando em um montante final de aproximadamente 1,0 t. Em seguida, realizou-se uma amostragem de 110 a $115 \mathrm{~kg}$ de resíduos, os quais foram segregados, adicionados sem compactação em recipientes identificados e pesados com o auxílio de uma balança eletrônica digital (Marca Filizola/Modelo MF 100), com capacidade máxima de $100 \mathrm{~kg}$ e sensibilidade de $0,02 \mathrm{~kg}$.

Como no Brasil não existe uma legislação específica que padronize a caracterização gravimétrica de RSD, foi utilizada a classificação da Norma Portuguesa, Portaria n 851/2009 (DIÁRIO DA REPÚBLICA PORTUGUESA, 2009, p. 5144), que é a mesma adotada pela Comunidade Econômica Europeia (CEE). As diretrizes dessa Norma foram adaptadas

Tabela 2 - Classificação da classe social.

\begin{tabular}{c|c|c|c}
$\begin{array}{c}\text { Classe } \\
\text { social }\end{array}$ & Grupo & $\begin{array}{c}\text { Renda per } \\
\text { capita (R } \$ \\
1,00)\end{array}$ & $\begin{array}{c}\text { Renda familiar } \\
-4 \text { pessoas } \\
\text { (R\$ } 1,00)\end{array}$ \\
\cline { 2 - 4 } & Extremamente pobre & O a 81 & O a 324 \\
\cline { 2 - 4 } Baixa & $\begin{array}{c}\text { Pobre, mas não } \\
\text { extremamente pobre }\end{array}$ & 82 a 162 & 325 a 648 \\
\cline { 2 - 4 } & Vulnerável & 163 a 291 & 649 a 1.164 \\
\cline { 2 - 4 } & Baixa classe media & 292 a 441 & 1.649 a 1.764 \\
\hline \multirow{2}{*}{ Média } & Média classe media & 442 a 641 & 1.765 a 2.564 \\
\cline { 2 - 4 } & Alta classe media & 642 a 1.019 & 2.565 a 4.076 \\
\hline \multirow{2}{*}{ Alta } & $\begin{array}{c}\text { Baixa classe alta } \\
\text { Alta classe alta }\end{array}$ & $\begin{array}{c}1.020 \text { a } 2.480 \\
\text { Acima de } \\
2.481\end{array}$ & $\begin{array}{c}4.077 \text { a } 9.920 \\
\text { Acima de } \\
9.921\end{array}$ \\
\hline
\end{tabular}

Fonte: Brasil (2012). à realidade brasileira. Assim, os resíduos foram qualificados em putrescíveis (somatório dos resíduos alimentares, de jardim e madeira/coco), recicláveis (composto pelos plásticos filme e rígido, metal, vidro, papel/ papelão, jornal/revista) e outros resíduos (soma dos componentes têxtil, sanitários, compósitos, borracha/couro, resíduos perigosos e rejeito), conforme adotado por Jucá et al. (2014). As informações relacionadas à predominância de estabelecimentos domiciliares e comerciais foram obtidas por meio de conhecimento prévio e visitas técnicas in loco.

\section{Seleção de indicadores e tratamento estatístico dos dados}

Recife é uma cidade de contrastes sociais, econômicos e ambientais aparentes, com perfis que versam desde áreas com características comerciais/empresariais a localidades fortemente verticalizadas ou com Zonas Especiais de Interesse Social (ZEIS), nas quais a pobreza é extrema. Dessa forma, a pesquisa contemplou alguns indicadores socioeconômicos e ambientais que representassem essa pluralidade, como, por exemplo: população; renda domiciliar e per capita; geração total e per capita de RSD; e composição gravimétrica: resíduos putrescíveis (resíduos alimentares, de jardim e madeira/coco) e recicláveis (plásticos, papel/papelão, metal e vidro). Assim, foi utilizada uma matriz de dimensão $31 \times 14$ referente às rotas de coleta e aos indicadores elencados no estudo. Os dados foram previamente padronizados por log $(\mathrm{x}+1)$, para que tivessem a mesma medida de escala, ficando graficamente mais bem representados, favorecendo a interpretabilidade das informações (FERRAZ, 2012; WANG et al., 2013).

No intuito de entender a inter-relação das rotas de coleta com os indicadores, foram utilizadas duas técnicas de análise multivariada: a análise de componente principal (ACP) e a análise de agrupamento hierárquico (AAH), ou análise por cluster. A análise multivariada é uma técnica que utiliza, simultaneamente, todas as variáveis na interpretação teórica dos dados obtidos. Segundo Hair et al. (2010), é uma técnica de análise quantitativa exploratória e não confirmatória dos dados, pois o tratamento das amostras em estudo gera perguntas/hipóteses, e não respostas/confirmações.

\section{Análise de componente principal}

A ACP, denominada por Hotelling (1933) de principal component analysis (PCA), é uma técnica estatística que possibilita a análise multivariada de dados, sem perdas significativas de informações. A análise consiste na conversão de uma matriz original $\mathrm{n} \times \mathrm{p}$ em uma matriz sintética (em que n é o número de indivíduos e p representa o conjunto de variáveis); na determinação de autovalores e autovetores; e na obtenção de combinações lineares das variáveis originais, as quais são denominadas de componentes principais (CP). As CP têm dimensão equivalente à matriz original, independência estatística, indivíduos similares agrupados e eliminação das variáveis originais com pouca informação (BRO; SMILDE, 2014). 
Cada combinação linear maximiza a variabilidade dos dados deixados pela matriz anterior. Assim, a primeira componente (CP1) apresentará maior grau de explicabilidade dos dados da matriz original, e a segunda componente (CP2) explicará o máximo da variância restante da CP1. A CP pode ser determinada por meio do algoritmo de variância-covariância $(\Sigma)$ ou de correlação (R) (SOARES et al., 2017). No presente estudo, utilizou-se a matriz de correlação para avaliar a inter-relação dos indicadores e identificar os mais representativos no estudo, como realizado por Agovino, Parodi e Sànchez Barajas (2014). Esses indicadores foram selecionados e utilizados na análise por cluster. O círculo unitário foi método gráfico adotado para a análise de correlação, conforme utilizado por Jardim et al. (2014).

\section{Análise de agrupamento hierárquico}

A AAH, ou agrupamento por cluster (NEDER, 2008), foi utilizada para agrupar 31 rotas de coleta com base nos indicadores mais representativos. De acordo com Manly (2008), a análise por cluster (cluster analysis) é uma técnica estatística multivariada que permite ao pesquisador gerar grupos mutuamente exclusivos por uma métrica de qualidade, com base em critérios predeterminados, de modo que tenham características de alta similaridade (homogeneidade) interna e elevada dissimilaridade (heterogeneidade) externa. Assim, a partir de uma matriz com n amostras (setores) e p variáveis (indicadores), procura-se um esquema de classificação que agrupe os objetos semelhantes em g grupos e subgrupos.

De acordo com Agovino, Ferrara e Garofalo (2016), a análise por cluster tem três principais etapas: definição da medida de distância entre as unidades estatísticas; transformação da matriz $(X)$ de dados $(n \times p)$ em uma matriz de diferenças ou distâncias $(n \times n)$ entre os $n$ pares de observações, sendo p elementos do vetor; e adoção de um algoritmo para agrupar as unidades estatísticas em grupos por similaridade.

A escolha da métrica de dissimilaridade depende da natureza dos dados analisados. Como o presente artigo utilizou dados quantitativos, foram empregadas medidas de distância. Existem diferentes medidas de distância, como Manhattan, Mahalanobis. Contudo, nesta pesquisa foi adotada a distância euclidiana como coeficiente de medida para mensurar as ligações entre os grupos formados ao longo dos passos de agrupamento, assim como foi realizado por Bari, Rosa e Pinto (2013).

Após essa etapa, o segundo passo consistiu na seleção do algoritmo de classificação e nos critérios de agregação. Os algoritmos de agrupamento podem ser hierárquicos ou não hierárquicos. Segundo Tumminello, Lillo e Mantegna (2010), ao utilizar o algoritmo hierárquico são testadas todas as possibilidades de agrupamento e é avaliada a quantidade de grupos mais pertinentes para a pesquisa. No entanto, no agrupamento não hierárquico, também conhecido como K-Means (HENNIG, 2008), não se produz diretamente uma partição em um número fixo de classes. No presente artigo, optou-se pelo método hierárquico para que fossem gerados grupos de rotas por similaridade.
Os clusters por similaridade gerados foram representados graficamente por meio de um dendrograma na forma horizontal, também conhecido como fenograma ou gráfico de árvore. Este foi utilizado horizontalmente para representar os agrupamentos das rotas por ordem decrescente de semelhança no eixo das ordenadas (eixo y) e as distâncias entre os grupos e subgrupos formados no eixo das abcissas (eixo $\mathrm{x}$ ).

O corte no dendrograma chamado de linha Fenon, que determina o número de grupos e subgrupos por similaridade gerados pela análise, seguiu o critério realizado por Jardim et al. (2014). Dessa forma, procedeu na região de maior salto do gráfico, ou seja, no ponto médio entre as maiores distâncias euclidianas dos grupos formados. A ACP e a AAH foram realizadas pelo programa Statistica versão 7.0.

\section{RESULTADOS E DISCUSSÃO}

\section{Análise de componentes principais dos indicadores}

A CP1 e a CP2 explicaram 70,20\% da variabilidade do conjunto de dados do modelo. Os indicadores população (POP), renda média domiciliar (RMD), renda média per capita (RMPC), geração de resíduos per capita (GRPC), resíduos recicláveis (RE), resíduos alimentares (RA) e resíduos putrescíveis (PU) foram bem representados no estudo, devido ao elevado peso dos autovalores (Figura 2). Ao passo que vidro (V), madeira/coco (MC) e geração de resíduos (GR) apresentaram dados com pouca informação, sendo excluídos da análise por cluster.

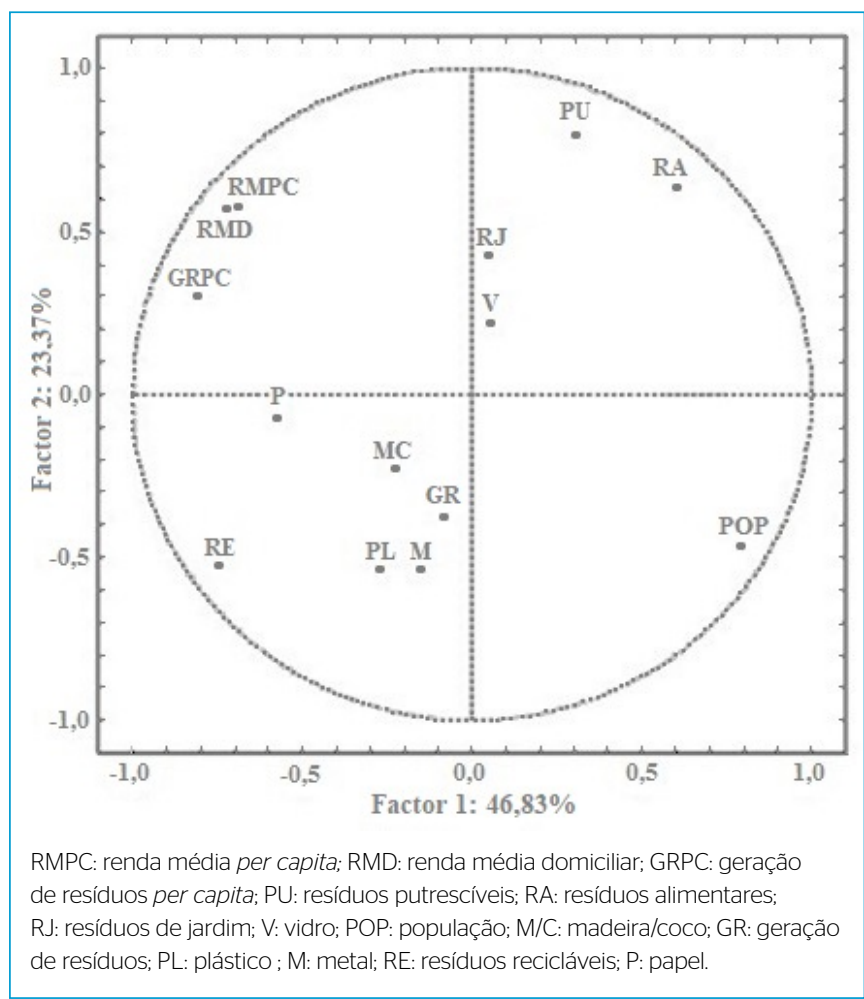

Figura 2 - Correlação dos 14 indicadores utilizados no estudo. 
Os indicadores renda (domiciliar e per capita) e geração per capita de resíduos apresentaram forte correlação positiva. Essa lógica foi constatada nos estudos realizados por Afroz, Hanaki e Tudin (2011), na cidade Dhaka, Bangladesh; por Godecke, Naime e Figueiredo (2012) e por Campos (2012), em algumas capitais brasileiras; por Ogwueleka (2013), na Nigéria; por Jadoon, Batool e Chaudhry (2014), em Lahore, no Paquistão; por Buenrostro-Delgado et al. (2015), em Morélia, no México; por Medeiros, Paz e Morais Júnior (2015), na cidade de João Pessoa; por Cardoso (2016), no Distrito Federal; e por Chu et al. (2016), na China.

No entanto, pesquisas realizadas por Dahlén et al. (2009), em 35 municípios da Suécia; por Getahun et al. (2012), em Jimma, na Etiópia; por Monavari et al. (2012), no Irã; por Saladié (2016), na Catalunha, Espanha; e por Xu et al. (2016), na ilha de Xiamen na China, não encontraram correlação positiva significativa entre essas variáveis. Segundo Xu et al. (2016), ainda não foi alcançada uma visão consensual quanto à correlação positiva entre renda e geração de RSD, pois essa relação depende de outros fatores, como, por exemplo, o grau de urbanização da área estudada. Nessa perspectiva, nos estágios iniciais de urbanização, a ascensão da renda familiar estimularia o consumo e, por consequência, aumentaria a geração de RSD. Contudo, os efeitos positivos do nível de renda sobre o consumo diminuiriam gradualmente conforme o processo de urbanização. Assim, nos estágios avançados, o aumento da renda implicaria redução da geração de RSD, devido à difusão da consciência ambiental da população. Isso foi observado pelos autores na Ilha de Xiamen, na China, em que constataram correlação não significativa da renda e geração de resíduos, em virtude dos estágios avançados de urbanização na área estudada.

A população das rotas selecionadas apresentou forte correlação negativa com a geração de resíduos per capita e com a renda. Assim, rotas de classe baixa e com elevada concentração populacional apresentaram baixos quantitativos de resíduos per capita. Essa lógica aplicou-se nas rotas de classe alta com baixa população, que apresentou elevada geração per capita de RSD. Notou-se que a relação renda e geração per capita com a composição gravimétrica dos RSD revelou dois eixos interpretativos:

- de independência - a tipologia dos resíduos não influencia fatores socioeconômicos, denominando-se como variáveis independentes;

- de dependência - a renda e a geração per capita dos RSD interferem na gravimetria dos resíduos sólidos gerados, inclusive em seus percentuais de recicláveis e putrescíveis.

\section{Análise de agrupamento hierárquico das rotas}

O dendrograma horizontal foi originado no salto de agrupamento 0,27 , entre os passos 29 e 30 de distância euclidiana de 0,80 e 1,07 (Figura 3). A linha Fenon foi traçada na distância de 0,90, gerando

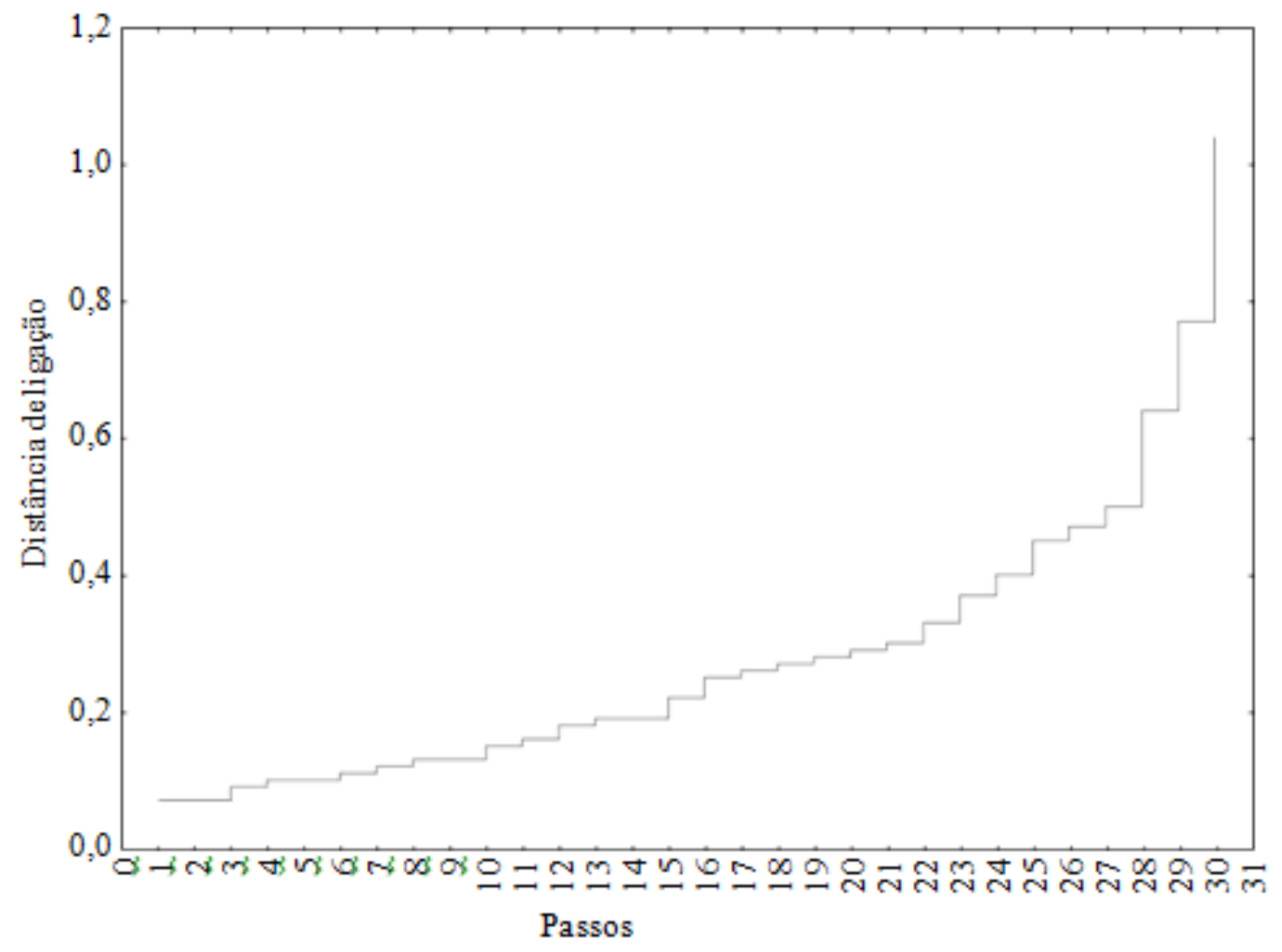

Figura 3 - Gráfico dos passos de agrupamentos e das distâncias euclidianas. 
dois clusters. O primeiro (Grupo A) deu origem a dois subgrupos bem definidos: A1 e A2; e o segundo (Grupo B), a três subgrupos: B1, B2 e B3 (Figuras 4 e 5). O grupo A compreendeu 17 rotas e o B abrangeu 14.

O subgrupo A1 compreendeu as rotas 1-04, 1-22, 1-30, 2-44, 2-14, 2-34, 6-82, 6-84, 3-37 e 4-17, e caracterizou-se por abranger bairros predominantemente de classe baixa que tendem à classe média, com alta concentração populacional, forte característica residencial e presença marcante de comércio formal e informal. Apresentou geração per capita média de RSD de $0,54 \mathrm{~kg} \cdot \mathrm{hab}^{-1} \cdot \mathrm{dia}^{-1}$ e maiores percentuais de resíduos recicláveis $(43,26 \%)$ diante dos putrescíveis $(32,77 \%)$.

O subgrupo A2 contemplou as rotas 3-01, 5-22V, 5-32V, 6-12, 6-20, 5-14 e 6-24. Caracterizou-se como um cluster de classe social muito baixa, com renda média domiciliar em torno de $\mathrm{R} \$ 587,84$ mês ${ }^{-1}$, e por um adensamento urbano informal altamente concentrado, denominado de favelas ou comunidades, com moradias precárias e miséria extrema. As rotas desse subgrupo localizaram-se nas regiões norte e nordeste da cidade do Recife, apresentaram baixa geração per capita de RSD $\left(0,44 \mathrm{~kg} \cdot \mathrm{hab}^{-1} \cdot \mathrm{dia}^{-1}\right)$ e percentuais bem equilibrados de resíduos putrescíveis $(37,41 \%)$ e recicláveis $(36,21 \%)$.

O subgrupo B1, formado pelas rotas 1-16, 3-05, 3-45, 3-09, 4-03 e 4-23, compreendeu os bairros de classe alta verticalizada do estudo,

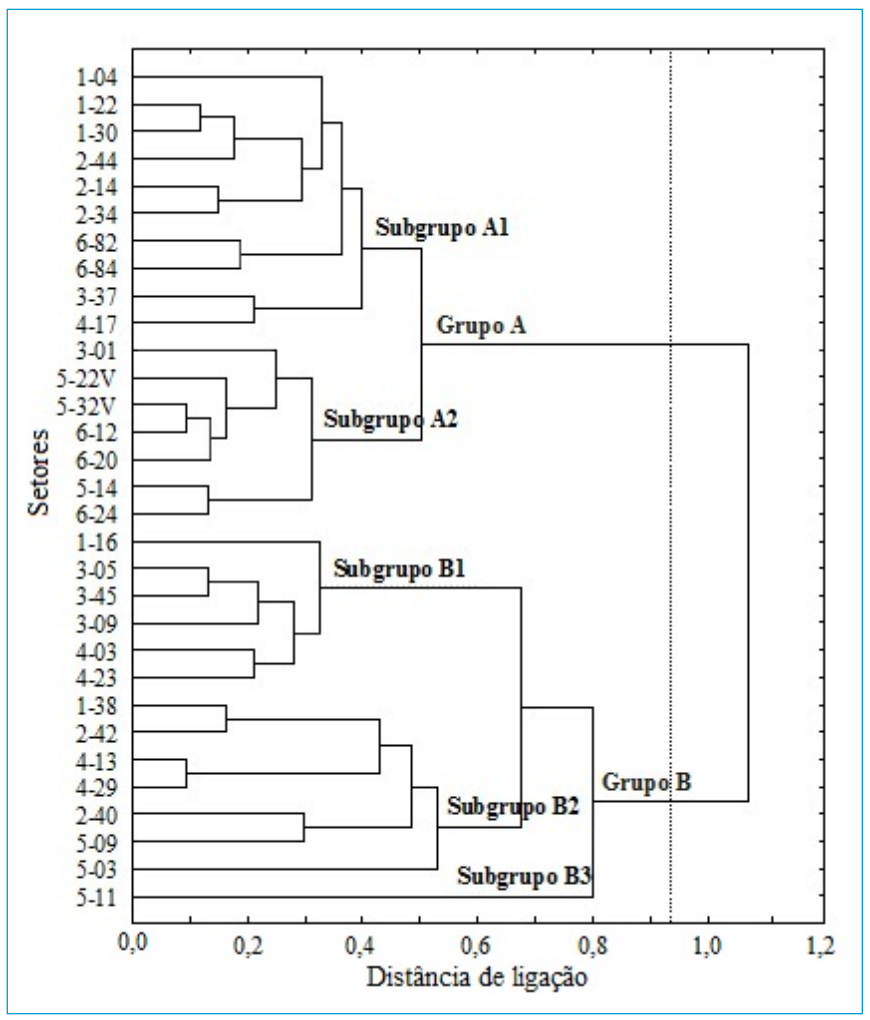

Figura 4 - Dendrograma dos clusters por similaridade das 31 rotas de coleta do Recife. com renda per capita mensal superior a R \$1.900. Desse modo, caracterizou-se como um cluster de alto poder aquisitivo, por abranger áreas privilegiadas da cidade, com constante ascensão econômica e valorização imobiliária. O cluster apresentou geração per capita média de $0,89 \mathrm{~kg} \cdot \mathrm{hab}^{-1} \cdot \mathrm{dia}^{-1}$ e altos percentuais de resíduos potencialmente recicláveis (45\%). Notou-se, além da coleta convencional, a presença de coleta seletiva porta a porta e de pontos de entrega voluntária (PEV) para os resíduos sólidos secos.

O subgrupo B2 compreendeu as rotas 1-38, 2-42, 4-13, 4-29, 2-40, 5-09 e 5-03, e caracterizou-se pela presença de bairros de classe média com baixa concentração populacional. O cluster compreendeu bairros com característica residencial e comercial com renda per capita mensal em torno de R\$ 740. Por contemplar áreas com forte atividade comercial, como os setores 5-03 e 5-09, o subgrupo apresentou geração per capita de RSD em torno de $1,00 \mathrm{~kg} \cdot \mathrm{hab}^{-1} \cdot \mathrm{dia}^{-1}$, além de maiores percentuais de resíduos

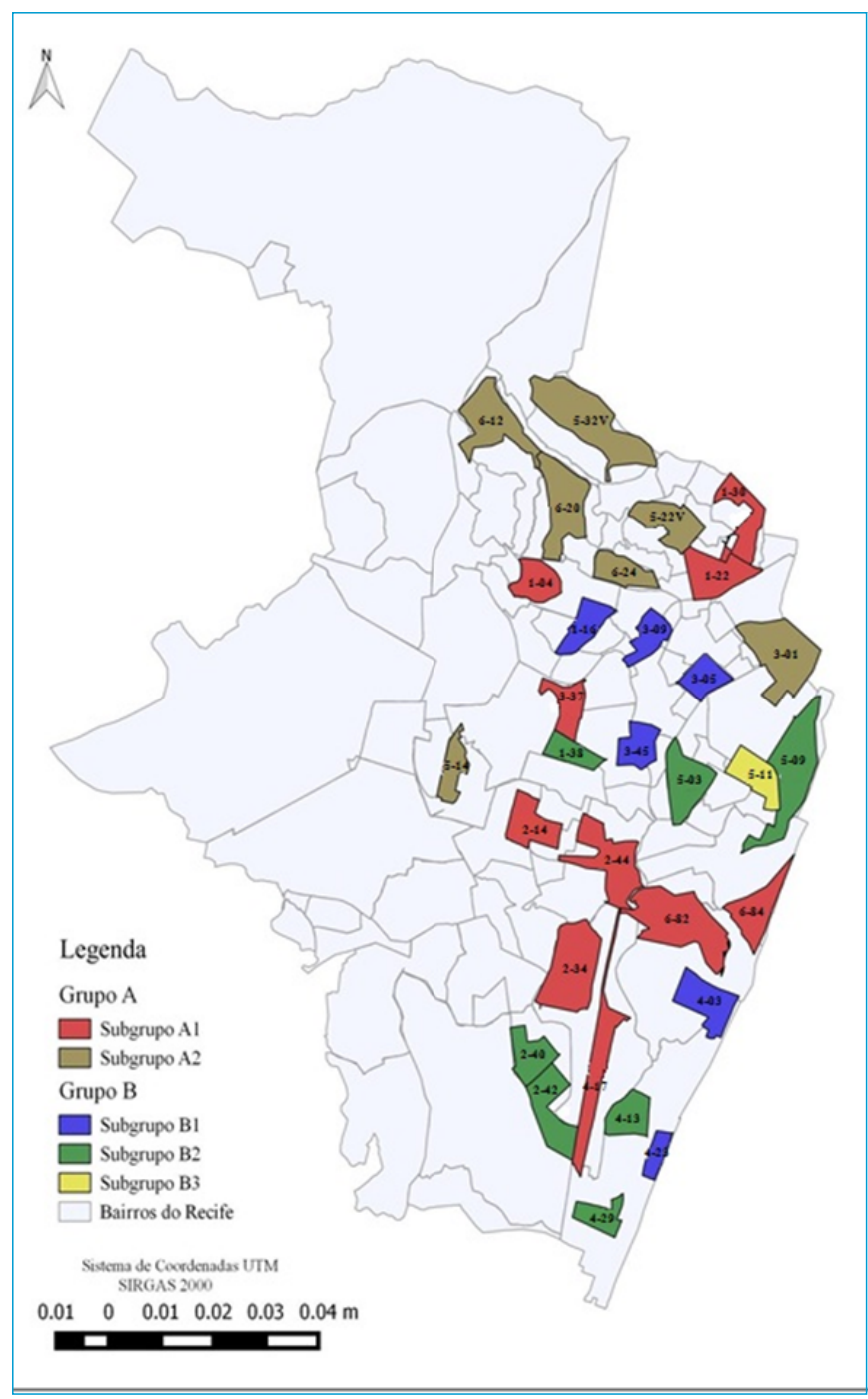

Figura 5 - Setorização dos clusters por similaridade das 31 rotas de coleta do Recife. 
potencialmente recicláveis $(41,36 \%)$ diante dos resíduos putrescíveis $(37,64 \%)$.

O subgrupo B3, diferente dos demais, contemplou apenas o setor 5-11, que corresponde aos bairros de Santo Amaro e Boa Vista. Essa particularidade foi decorrente da elevada geração per capita de RSD na rota, com valor aproximado de 3,20 kg.hab ${ }^{-1} \cdot \mathrm{dia}^{-1}$, sendo reflexo do forte e difundido comércio realizado nessas áreas. Dessa forma, caracterizou-se como um cluster de classe média, com renda per capita em torno de $\mathrm{R} \$ 900$, essencialmente comercial e com população flutuante. Além disso, apontou percentuais bem equilibrados de resíduos recicláveis $(45,56 \%)$ e putrescíveis $(43,43 \%)$, o que diferiu das demais rotas comerciais do estudo.

Se observadas as características dos grupos e subgrupos formados pelas similaridades das rotas de coleta estudadas (Tabela 3), nota-se que há relação entre renda, geração per capita e recicláveis, tendo as classes mais favorecidas valores desses itens maiores também. Por outro lado, esses clusters apontam perfis de rotas de coleta domiciliar da cidade do Recife que poderão auxiliar o gestor do serviço na estruturação de modelos setoriais heterogêneos de RSD que busquem as particularidades das áreas estudadas, sendo uma possibilidade de ferramenta para a tomada de decisão no sentido de elevar a eficiência e a eficácia do processo de coleta de recicláveis.

Alguns pesquisadores também utilizaram a análise por cluster para setorizar áreas a partir de indicadores. Silva, Barbieri e Monte-Mór (2012) obtiveram sete clusters que agruparam domicílios do município de Belo Horizonte (MG) com base nas variáveis: renda, educação, estrutura etária e domiciliar, e geração de RSD. Bari, Mata e Wanderley (2014) obtiveram dois clusters para agrupar 27 estados do Brasil com base no grau de adequação do sistema de gestão. Cardoso (2016) verificou a formação de três clusters para a geração de RSD de 2003 a 2014 do Distrito Federal. Agovino, Ferrara e Garofalo (2016) constataram o surgimento de dois clusters que agruparam 103 províncias italianas com base na adequação do sistema de gerenciamento de RSD. Sulkowski e White (2016) verificaram dois clusters que contemplaram 61 países mediante indicadores de felicidade, desenvolvimento, renda e emissão de carbono. Lima et al. (2017) encontraram quarto clusters de países por similaridade quanto ao PIB, ao desenvolvimento sustentável e ao índice de competitividade. Já Costa (2017) obteve cinco clusters formados por 31 rotas de coleta de RSD do Recife com base na pegada ecológica da fração seca reciclável. Entretanto, essa tecnologia de análise ainda não foi plenamente internalizada como um procedimento para a definição das estratégias e dos modelos de coleta de resíduos sólidos, especialmente dos recicláveis, havendo vasto campo para que tais procedimentos sejam compreendidos plenamente pela gestão pública.

\section{CONCLUSÃO}

Neste trabalho foram utilizadas técnicas estatísticas multivariadas para setorizar 31 rotas de coleta de RSD da cidade do Recife, a partir da identificação dos indicadores mais representativos e do grau de similaridade dos clusters. A população, a renda domiciliar e per capita, a geração de resíduos per capita e os resíduos recicláveis, alimentares e putrescíveis foram bem representados no modelo. Já o vidro, a madeira/coco e a geração de resíduos (GR) apresentaram dados com pouca informação. A renda e a geração per capita apresentaram forte correlação positiva, em detrimento da correlação negativa com a população. Assim, a renda apresentou-se como o principal indicador para geração de RSD produzidos nas rotas do Recife.

As 31 rotas de coleta foram setorizadas em dois grupos (A e B), que resultaram em cinco subgrupos por similaridade (A1, A2, B1, B2 e B3). Os subgrupos apresentaram características socioeconômicas e ambientais distintas. Os subgrupos A1 e A2 compreenderam setores de classe baixa, ao passo que os clusters B1, B2 e B3, os setores de classe alta, média e comercial, respectivamente. A setorização das rotas mediante o grau de similaridade dos clusters mostrou-se uma ferramenta interessante de análise, principalmente como etapa para a estruturação de modelos setoriais heterogêneos de resíduos sólidos. Tais procedimentos analíticos deveriam ser considerados como ferramenta auxiliar na tomada de decisão em relação à gestão pública dos RSD.

Tabela 3 - Resumo das características dos clusters por similaridade.

\begin{tabular}{|c|c|c|c|c|c|c|c|c|c|c|c|}
\hline G & SG & Rotas & POP & DD & CE & RMD & RMPC & GR & GRPC & RE & PU \\
\hline \multirow{2}{*}{ A } & A1 & 10 & 16.459 & 164,26 & Baixa & $1.046,50$ & 318,68 & 255,50 & 0,54 & 43,26 & 32,77 \\
\hline & A2 & 7 & 23.830 & 224,31 & Baixa & 587,84 & 169,59 & 301,62 & 0,44 & 36,21 & 37,41 \\
\hline \multirow{3}{*}{ B } & B1 & 6 & 9.565 & 176,28 & Alta & $5.490,41$ & $1.907,56$ & 249,40 & 0,89 & 42,54 & 36,69 \\
\hline & B2 & 7 & 9.024 & 129,92 & Média & $2.165,05$ & 737,37 & 249,76 & 0,99 & 41,36 & 37,64 \\
\hline & B3 & 1 & 3.926 & 53,53 & Média & $2.146,67$ & 901,10 & 373,95 & 3,17 & 45,56 & 43,43 \\
\hline
\end{tabular}

G: grupos; SG: subgrupos; POP: população (n. hab.); DD: densidade demográfica (hab.ha'1); CE: classe econômica; RMD: renda média domiciliar (R\$.mês'); RMPC: renda mensal per capita; GR: geração média de resíduos por mês (ton.mês'); GRPC: geração de resíduos per capita (kg.hab.'.'dia'); RE: recicláveis (\%); PU: putrescíveis (\%). 


\section{REFERÊNCIAS}

AFROZ, R.; HANAKI, K.; TUDIN, R. (2011) Factors affecting waste generation: a study in waste management program in Dhaka City, Bangladesh. Environmental Monitoring Assessment, v. 179, p. 509519. https://doi.org/10.1007/s10661-010-1753-4

AGOVINO, M.; FERRARA, M.; GAROFALO, A. (2016) An exploratory analysis on waste management in Italy: A focus on waste disposed in landfill. Land Use Policy, v. 57, p. 669-681. https://doi.org/10.1016/j. landusepol.2016.06.027

AGOVINO, M.; PARODI, G.; SÀNCHEZ BARAJAS, G. (2014) An analysis of disabled peopleand the labor market in Mexico. Latin America Research Review, v. 49, n. 2, p. 221-242. https://doi.org/10.1353/lar.2014.0035

ASSOCIAÇÃO BRASILEIRA DE NORMAS TÉCNICAS (ABNT). (2OO4) NBR 10007: Amostragem de resíduos. Rio de Janeiro: ABNT. Disponível em: <https://wp.ufpel.edu.br/residuos/files/2014/O4/nbr10007-amostragem-de-resc3adduos-sc3b3lidos.pdf>. Acesso em: 8 nov. 2017.

BARI, M.L.; MATA, H.T.C.; WANDERLEY, L.A. (2014) Gestão de resíduos nos estados/municípios brasileiros: avaliação e análise dos procedimentos para a escolha do modelo de gestão ambiental. Nexos Econômicos, v. 8, n. 2, p. 107-121.

BARI, M.L.; ROSA, M.J.A.; PINTO, A.C. (2013) Gestão de resíduos nos estados/municípios brasileiros: avaliação e análise dos procedimentos para a escolha do modelo de gestão ambiental. In: ENCONTRO DA SOCIEDADE BRASILEIRA DE ECONOMIA ECOLÓGICA, 10., 2013. Anais... Vitória.

BRASIL. (2010) Lei 12.305, de 2 de agosto de 2010. Institui a Política Nacional de Resíduos Sólidos. Disponível em: <http://www.planalto. gov.br/ccivil_03/_ato2007-2010/2010/lei//12305.htm>. Acesso em: 8 nov. 2017.

BRASIL. (2012). Secretaria de Assuntos Estratégicos (SAE). Presidência da República. Relatório de definição da classe média no Brasil. São Paulo: Secretaria de Assuntos Estratégicos. Disponível em: <https://silo.tips/download/definiao-da-classe-media-no-brasilsao-paulo-junho-de-2012>. Acesso em: 13 out. 2020.

BRO, R.; SMILDE, A.K. (2014) Principal component analysis. Analytical Methods, v. 6, p. 2812-2831. https://doi.org/10.1039/C3AY41907J

BUENROSTRO-DELGADO, O.; ORTEGA-RODRIGUEZ, J.M.; CLEMITSHAW, K.C.; GONZÁLEZ-RAZO, C:; HERNÁNDEZ-PANIAGUA, I.Y. (2015) Use of genetic algorithms to improve the solid waste collection service in an urban área. Waste Management, v. 41, p. 20-27. https://doi.org/10.1016/j.wasman.2015.03.026

CAMPOS, H.K.T. (2012) Renda e evolução da geração per capta de resíduos sólidos no Brasil. Engenharia Sanitária e Ambiental, v. 17, n. 2, p. 171-180. http://dx.doi.org/10.1590/S1413-41522012000200006

CARDOSO, M.S.S. (2016) A gestão de resíduos sólidos urbanos na RIDE-DF: a geração e a coleta seletiva. Dissertação (Mestrado em Desenvolvimento Sustentável) - Universidade de Brasília, Brasília.
CARIBÉ, R.M. (2015) Análise multivariada no comportamento dos recalques em célula experimental de resíduos sólidos urbanos. Dissertação (Mestrado em Engenharia Civil e Ambiental) Universidade Federal de Campina Grande, Campina Grande.

CHASSAIGNE, G.; PINTO, G. (2014) Determinación de variables que inciden en la estimación de residuos y desechos sólidos municipales recolectados en Venezuela. Interciência, v. 39, n. 12, 874-881.

CHU, Z.; WU, Y.; ZHOU, A.; HUANG, W.C. (2016) Analysis of influence factors on municipal solid waste generation based on the multivariable adjustment. Environmental Progress \& Sustainable Energy, v. 35, n. 6, p. 1629-1633. http://doi.org/10.1002/ep.12399

COSTA, A.R.S. (2017) Análise da gestão dos resíduos sólidos urbanos da cidade de Recife-PE a partir de indicadores de sustentabilidade. Dissertação (Mestrado em Engenharia Ambiental) - Universidade Federal Rural de Pernambuco, Recife.

DAHLÉN, L.; BERG, H.; LAGERKVIST, A.; BERG, P.E.O. (2009) Inconsistent pathways of household waste. Waste Management, $\mathrm{v}$. 29, n. 6, p. 1798-1806. https://doi.org/10.1016/j.wasman.2008.12.004

DIÁRIO DA REPÚBLICA PORTUGUESA. (2009) Portaria no 851/2009. Norma portuguesa que estabeleci a padronização da caracterização gravimétrica dos resíduos sólidos. 1a Série, n. 152.

FERRAZ, H.D.A. (2012) Associação da Ocorrência de Cianobactérias às Variações de Parâmetros de Qualidade da Água em Quatro Bacias Hidrográficas de Minas Gerais. 133f. Dissertação (Mestrado em Saneamento, Meio Ambiente e Recursos Hídricos) Universidade Federal de Minas Gerais, Belo Horizonte.

FUDALA-KSIAZEK, S.; PIERPAOLI, M.; KULBAT, E.; LUCZKIEWICZ, A. (2016) A modern solid waste management strategy - the generation of new by-products. Waste Management, v. 49, p. 516529. https://doi.org/10.1016/j.wasman.2016.01.022

GETAHUN, T.; MENGISTIE, E.; HADDIS, A.; WASIE, F.; ALEMAYEHU, E. DADI, D.; VAN GERVEN, T.; VAN DER BRUGGEN, B. (2012) Municipal solid waste generation in growing urban areas in Africa: current practices and relation to socioeconomic factors in Jimma, Ethiopia. Environmental Monitoring Assessment, v. 184, n. 10, p. 6337-6345. https://doi.org/10.1007/s10661-011-2423-x

GODECKE, M.V.; NAIME, R.H.; FIGUEIREDO, J.A.S. (2012) O consumismo e a geração de resíduos sólidos no Brasil. Revista Gestão, Educação e Tecnologia Ambiental, v. 8, n. 8, p. 1700-1712. http://dx.doi.org/10.5902/223611706380

HAIR, J.F.; BLACK, W.C.; BABIN, B.J.; ANDERSON, R.E. (2010) Multivariate Data Analysis. 7. ed. Nova York: Pearson.

HENNIG, C. (2008) Dissolution point and isolation robustness: robustness criteria for general cluster analysis methods. Journal of Multivariate Analysis, v. 99, n. 6, p. 1154-1176. https://doi.org/10.1016/j. jmva.2007.07.002 
HOTELLING, H. (1933) Analysis of a Complex of Statistical Variables into Principal Components. Journal of Educational Psychology, v. 24, n. 6, p. 417-441. https://psycnet.apa.org/doi/10.1037/h0071325

INSTITUTO BRASILEIRO DE GEOGRAFIA E ESTATISTICA (IBGE). (2010) Censo demográfico 2010: resultados da amostra rendimento para os municípios pernambucanos. Brasil: IBGE.

INSTITUTO BRASILEIRO DE GEOGRAFIA E ESTATÍSTICA (IBGE). (2016) Estimativa dos dados de Recife 2016. Brasil: IBGE. Disponível em: <http://cidades.ibge.gov.br/xtras/perfil.php?codmun=261160>. Acesso em: 12 ago. 2017

JADOON, A.; BATOOL, S.A.; CHAUDHRY, M.N. (2014) Assessment of factors affecting household solid waste generation and its composition in Gulberg Town, Lahore, Pakistan. Journal of Material Cycles and Waste Management, v. 16, n. 1, p. 73-81. https://doi. org/10.1007/s10163-013-0146-5

JARDIM, F.A.; VON SPERLING, E.; JARDIM, B.F.M.; ALMEIDA, K.C.B. (2014) Fatores determinantes das florações de cianobactérias na água do Rio Doce, Minas Gerais, Brasil. Revista Engenharia Sanitária e Ambiental, v. 19, n. 3, p. 207-218. https://doi.org/10.1590/ S1413-41522014019000001026

JUCÁ, J.F.T. FIRMO, A.L.B.; OLIVEIRA, L.R.G.; BRITO, E.P.L.; SILVA, R.C.P; GÓlS, T.M.L. (2014) Estudo da geração e composição dos resíduos sólidos urbanos da cidade de Recife. Relatório técnico. Versão final. Recife.

LENZ, S.; BÖHM, K.; OTTNER, R.; HUBER-HUMER, M. (2O16) Determination of leachate compounds relevant for landfill aftercare using FT-IR spectroscopy. Waste Management, v. 55, p. 321-329. https://doi.org/10.1016/j.wasman.2016.02.034

LIMA, M.L.B.; FROTA, J.A.; ARAÚJO, F.S.M.; FONTENELE, R.E.S. (2O17) Desenvolvimento sustentável e competitividade das nações: uma análise multivariada. REUNA, v. 22, n. 1, p. 41-62. https://doi. org/10.21714/2179-8834/2017v22n1p41-62

MANLY, B.J.F. (2008) Métodos estatísticos multivariados: Uma introdução. 3. ed. Porto Alegre: Bookman. 230 p.

MEDEIROS, J.E.S.F.; PAZ, A.R.; MORAIS JÚNIOR, J.A. (2O15) Análise da evolução e estimativa futura da massa coletada de resíduos sólidos domiciliares no município de João Pessoa e relação com outros indicadores de consumo. Engenharia Sanitária e Ambiental, v. 20, n. 1, p. 119-130. https://doi.org/10.1590/S1413-41522015020000120117

MONAVARI, S.M.; OMRANI, G.A.; KARBASSI, A.; RAOF, F.F. (2O12) The effects of socioeconomic parameters on household solid-waste generation and composition in developing countries (a case study: Ahvaz, Iran). Environmental Monitoring Assessment, v. 184, p. 18411846. https://doi.org/10.1007/s10661-011-2082-y

NEDER, H.D. (2008) Estrutura do mercado de trabalho agrícola no Brasil: uma análise descritiva da evolução de suas categorias entre 1995 e 2006. In: BUAINAIN, A.M.; DEDECCA, C. (Org.). Emprego e trabalho na agricultura brasileira. Brasília: IICA. p. 156-182. Disponível em: <https://ainfo.cnptia.embrapa.br/digital/bitstream/item/35341/1/ Serie-DRS-vol-9-Emprego-e-Trabalho-na-Agricultura-Brasileira.pdf>. Acesso em: 13 out. 2020.
NI, Z.; LIU, J.; ZHANG, M. (2017) Short-term pre-aeration applied to the dry anaerobic digestion of MSW, with a focus on the spectroscopic characteristics of dissolved organic matter. Chemical Engineering Journal, v. 313, 1222-1232. https://doi. org/10.1016/j.cej.2016.11.020

OGWUELEKA, T.C. (2013) Survey of household waste composition and quantities in Abuja, Nigeria. Resources, Conservation and Recycling, v. 77, p. 52-60. https://doi.org/10.1016/j. resconrec.2013.05.011

PERNAMBUCO. (2010) Lei no 14.236, de 13 de dezembro de 2010 Dispõe sobre a Política Estadual de Resíduos Sólidos, e dá outras providências. Pernambuco. Disponível em: <http://www.cprh. pe.gov.br/ARQUIVOS_ANEXO/Lei\%2014236;141010;20101229.pdf> Acesso em: 8 nov. 2017.

PURCELL, M.; MAGETTE, W.L. (2009) Prediction of household and commercial BMW generation according to socioeconomic and other factors for the Dublin region. Waste Management, v. 29, n. 4, p. 1237-1250. https://doi.org/10.1016/j. wasman.2008.10.011

RECIFE. (2014) Plano de gestão integrada de resíduos sólidos urbanos de Recife (PGIRS). Diagnóstico e análise da situação atual (Produto 2). Recife: BRENCORP - Consultoria, Meio Ambiente e Empreendimento.

REGO, R.F.; LIMA, V.C.; LIMA, A.C.; BARRETO, M.L.; PRADO, M.S.; STRINA, A. (2013) Environmental indicators of intra-urban hetererogeneity. Caderno de Saúde Pública, v. 29, n. 6, p. 1173-1185. https://doi.org/10.1590/S0102-311X2013000600014

SALADIÉ, O. (2016) Determinants of waste generation per capita in Catalonia (North-eastern Spain): the role of seasonal population. European Journal of Sustainable Development, v. 5, n. 3, p. 489-504. https://doi.org/10.14207/ejsd.2016.v5n3p489

SCHIRMER, W.N.; CROVADOR, M.I.C.; MARTINS, K.G.; FRANQUETO, R.; CABRAL, A.R.; CAPANEMA, M.A.; JUCÁ, J.F.T. (2016) Análise por componentes principais para o volume de biogás gerado em aterro sanitário e a correlação com variáveis físico-químicas. Revista Gestão Sustentável e Ambiental, v. 5, n. 2, p. 149-164. http:// dx.doi.org/10.19177/rgsa.v5e22016149-164

SECRETARIA DE DESENVOLVIMENTO URBANO E HABITAÇÃO (SECID). Secretaria das Cidades. (2015) Consórcio metropolitano de resíduos sólidos. SECID. Disponível em: <http://www.portais. pe.gov.br/web/secid/programas-acoes/consorcio-metropolitanode-residuos-solidos>. Acesso em: 8 nov. 2017.

SEL, I.; ÇAKMAKCI, M.; ÖZKAYA, B.; ALTAN, H.S. (2016) Case study on prediction of remaining methane potential of landfilled municipal solid waste by statistical analysis of waste composition data. Waste Management, v. 56, p. 310-317. https://doi.org/10.1016/j. wasman.2016.07.023

SILVA, H.; BARBIERI, A.F.; MONTE-MÓR, R.L. (2012) Demografia do consumo urbano: um estudo sobre a geração de resíduos sólidos domiciliares no município de Belo Horizonte. Revista Brasileira de Estatística Populacional, v. 29, n. 2, p. 421-449. https://doi.org/10.1590/ S0102-30982012000200012 
SISTEMA NACIONAL DE INFORMAÇÕES SOBRE SANEAMENTO (SNIS). Diagnóstico do manejo de resíduos sólidos urbanos - 2017. Disponível em:< http://snis.gov.br/diagnostico-residuos-solidos/ diagnostico-rs-2017>. Acesso em: O3 jun. 2019.

SOARES, M.A.R.; QUINA, M.J.; REIS, M.S.; QUINTA-FERREIRA, R. (2017) Assessment of co-composting process with high load of an inorganic industrial waste. Waste Management, v. 59, p 80-89. https://doi.org/10.1016/j.wasman.2016.09.044

SULKOWSKI, A.; WHITE, D.S. (2016) A happiness Kuznets curve? Using model-based cluster analysis to group countries based on happiness, development, income, and carbon emissions. Environmental Development Sustainable, v. 18, p. 1095-1111. https:/l doi.org/10.1007/s10668-015-9689-z
TUMMINELLO, M.; LILLO, F.; MANTEGNA, R.N. (2010) Correlation, hierarchies, and networks in financial markets. Journal of Economic Behavior \& Organization, v. 75, n. 1, p. 40-58. https://doi.org/10.1016/j. jebo.2010.01.004

XU, L.; LIN, T.; XU, Y.; XIAO, L.; YE, Z.; CUI, S. (2016) Path analysis of factors influencing household solid waste generation: a case study of Xiamen Island, China. Journal of Material Cycles Waste Management, v. 18, p. 377-384. https://doi.org/10.1007/ s10163-014-0340-0

WANG, H.; SHANGGUAN, L.; WU, J.; GUAN, R. (2013) Multiple linear regression modeling for compositional data. Neurocomputing, v. 122, p. 490-500. https://doi.org/10.1016/j. neucom.2013.05.025 